\title{
hsa-miR-15a-5p inhibits colon cell carcinoma via targeting CCND1
}

\author{
ZHIPENG LI ${ }^{1 *}$, ZEYU ZHU $^{2 *}$, YANJUN WANG $^{3}$, YING WANG $^{4}$, WEIBING LI ${ }^{1}$, \\ ZHIGANG WANG $^{5}$, XINYUAN ZHOU $^{6}$ and YUHUA BAO ${ }^{1}$ \\ ${ }^{1}$ Department of Integrated Traditional Chinese and Western Medicine, Jiangsu Cancer Hospital and Jiangsu Institute of \\ Cancer Research and The Affiliated Cancer Hospital of Nanjing Medical University, Nanjing, Jiangsu 210009; \\ ${ }^{2}$ Department of Orthopedics, Huaian Hospital, Huaian, Jiangsu 223200; ${ }^{3}$ Department of Women's Preventive Health, \\ Huishan No. 2 People's Hospital, Wuxi, Jiangsu 214400; Departments of ${ }^{4}$ Radiotherapy and ${ }^{5}$ Hospital Quality Management, \\ Jiangsu Cancer Hospital and Jiangsu Institute of Cancer Research and The Affiliated Cancer Hospital of \\ Nanjing Medical University, Nanjing, Jiangsu 210009; ${ }^{6}$ Department of Gastroenterology, \\ Wuxi Hospital of TCM, Affiliated to Nanjing University of Chinese Medicine, Wuxi, Jiangsu 214000, P.R. China
}

Received May 20, 2020; Accepted February 9, 2021

DOI: $10.3892 / \mathrm{mmr} .2021 .12375$

\begin{abstract}
Colon carcinoma is one of the most common cancers worldwide. Epidemiological studies have revealed that colon cancer is the third leading cause of cancer-related deaths, which is due to the increased incidence and mortality rates. However, the treatment strategies for colon cancer remain unsatisfactory for patients, especially for those with advanced or recurrent colon cancer. Dysregulated microRNAs (miRNAs) are considered to influence tumor development and metastasis. However, the molecular mechanism through which miRNAs affect cancer progression is not yet completely understood. The aim of the present study was to investigate the expression levels of has-miR-15a-5p and its molecular mechanism in colon cell carcinoma. In the present study, the expression levels of hsa-miR-15a-5p were found to be decreased in colon tumor tissues and cancer cell lines. Hsa-miR-15a-5p
\end{abstract}

Correspondence to: Dr Yuhua Bao, Department of Integrated Traditional Chinese and Western Medicine, Jiangsu Cancer Hospital and Jiangsu Institute of Cancer Research and The Affiliated Cancer Hospital of Nanjing Medical University, 42 Baiziting, Xuanwu, Nanjing, Jiangsu 210009, P.R. China

E-mail: jszlyybyh@163.com

Dr Xinyuan Zhou, Department of Gastroenterology, Wuxi Hospital of TCM, Affiliated to Nanjing University of Chinese Medicine, 8 Zhongnan West Road, Binhu, Wuxi, Jiangsu 214000, P.R. China

E-mail: zxy403832760@sina.com

${ }^{*}$ Contributed equally

Abbreviations: miRNA/miR, microRNA; CCND1, G1/S-specific cyclin-D1; RT-qPCR, reverse transcription-quantitative PCR; CCK-8, Cell Counting Kit-8; EdU, 5-ethynyl-2'-deoxyuridine

Key words: colon cancer, hsa-microRNA-15a-5p, G1/S-specific cyclin-D1 overexpression inhibited colon cell proliferation and migration. Mechanistically, the G1/S-specific cyclin-D1 (CCND1) gene was predicted as a target of hsa-miR-15a-5p, as evidenced by bioinformatics and dual-luciferase reporter assay analyses. CCND1 overexpression significantly increased the progression of colon cancer. Furthermore, CCND1 was demonstrated to mediate the effects of hsa-miR-15a-5p on colon cancer cells. The present study demonstrated that hsa-miR-15a-5p alleviated the proliferation, migration and invasion of colon cancer by targeting the CCND1 gene, which represents a potential molecular target for the diagnosis and treatment of colon cancer.

\section{Introduction}

Colon carcinoma is a common digestive systemic sarcoma, with increasing incidence each year. According to the global cancer statistics in 2018, colon carcinoma has become the fourth leading cause of tumor-related deaths (1). Despite apparent curative surgery and chemotherapy, the mortality of colon carcinoma remains high, leading to poor five-year survival rates (2-5). Although dietary habits and living environments are improving, the onset age is shifting towards the younger population (6). Hence, patients succumb to colon carcinoma due to the rapid progression of this disease and its frequent recurrent metastasis. However, the pathological mechanism of colon cancer remains unclear. Thus, it is urgent to discover a pathogenic target gene that facilitates this disease.

Multiple factors, including oncogenes and tumor suppressors, are involved in tumorigenesis and metastasis. Among which, the irregular function of microRNAs (miRs/miRNAs) has been demonstrated to play an important role in cancer pathogenesis (7-9). miRNAs are highly conserved non-coding RNA molecules consisting of 20-24 nucleotides. miRNAs affect mRNA degradation or expression by completely or incompletely binding to the 3'-untranslated region (3'-UTR) of target genes. In addition, various miRNAs have been 
reported to modulate colon cancer progression (10-12). For example, miR-487b-3p has been found to inhibit colon cancer progression through directly targeting metabotropic glutamate receptor 3 (GRM3), thus indicating that the miR-487b-3p/GRM3/TGF- $\beta$ signaling axis is an important regulator of colon cancer tumorigenesis (13). Meanwhile, miR-524-5p was also reported to repress angiogenesis in colon cancer cells via targeting serine/threonine-protein kinase WNK1 (14). Of note, hsa-miR-15a-5p has been identified to be downregulated in colon cancer (15). However, its biological role and potential mechanism in colon cancer remains to be elucidated.

The present study aimed to identify the key functions of has-miR-15a-5p in colon cell carcinoma and demonstrated that hsa-miR-15a-5p targeted G1/S-specific cyclin-D1 (CCND1) and functioned as a tumor suppressor of colon cancer progression in vitro, as evidenced by the reduced proliferation, migration and invasion in colon cancer cells with overexpressed hsa-miR-15a-5p. The present findings provided a novel mechanism via which hsa-miR-15a-5p regulated colon cancer progression, thus hsa-miR-15a-5p could be a promising target for colon cancer diagnosis and therapy.

\section{Materials and methods}

Tissue samples. A total of 30 colon cancer and paired adjacent normal tissues ( $>5 \mathrm{~cm}$ from cancer tissue) were obtained from patients (18 male patients and 12 female patients) undergoing surgery between March 2017 and December 2018 at Jiangsu Cancer Hospital (Nanjing, China). The patients were aged between 42 and 65 years. Among them, the median age of male patients was 53.4 years (range, $42-63$ years), while the median age of female patients was 51.3 years (range, 45-65 years). The inclusion criteria were as follows: None of the patients received antitumor therapy, such as radiotherapy or chemotherapy, before surgery and final diagnosis was confirmed by routine pathological examination. The exclusion criteria were as follows: Patients who received pre-operative radiotherapy or chemotherapy. All specimens were frozen and conserved in liquid nitrogen at $-80^{\circ} \mathrm{C}$. Written informed consent was obtained from all patients before surgery. The present study was approved by the Ethics Committee of the Affiliated Cancer Hospital of Nanjing Medical University and Jiangsu Cancer Hospital and Jiangsu Institute of Cancer Research [(2016) approval no. 231].

Cell culture and transfection. Human colon cancer cell lines, SW480 and HCT116, the human colorectal cancer cell line, HT29, and human normal colonic epithelial cells, NCM460, were obtained from the American Type Culture Collection. Human colon cancer cell lines, SW480 and HCT116, were derived from patients with colon cancer. The human colorectal cancer cell line,HT29, was derived from patients with colorectal adenocarcinoma and HT29 cell line was authenticated by STR profiling before distribution. All cells were incubated in DMEM (cat. no. 319-005-CL; Wisent, Inc.) supplemented with 10\% FBS (Gibco; Thermo Fisher Scientific, Inc.), $100 \mathrm{U} / \mathrm{ml}$ penicillin and $100 \mu \mathrm{g} / \mathrm{ml}$ streptomycin in a humid atmosphere at $37^{\circ} \mathrm{C}$ in $5 \% \mathrm{CO}_{2}$. miRNA mimics (hsa-miR-15a-5p), miRNA inhibitors (hsa-miR-15a-5p), negative control mimics
(NC mimics) and miRNA negative control inhibitors (NC inhibitors) were purchased from Guangzhou RiboBio Co., Ltd. Among them, non-targeting $\mathrm{NC}$ mimics and $\mathrm{NC}$ inhibitors were used as the controls. HT29 cell lines were seeded on 6-well plates $\left(1 \times 10^{5}\right.$ cells/well) one day prior to transfection. The synthetic oligonucleotides or plasmids were used at $50 \mathrm{nmol} / \mathrm{ml}$ for transfection. Transfection was performed with Lipofectamine ${ }^{\circledR} 2000$ (Invitrogen; Thermo Fisher Scientific, Inc.) at $37^{\circ} \mathrm{C}$ according to the manufacturer's instructions. The cells were harvested 24-48 (for miRNA and mRNA expression) or 48-72 h (for protein expression) after transfection for functional assays or RNA/protein extraction. The sequences were as follows: hsa-miR-15a-5p mimics, 5'-UAGCAGAUC CCAAUGGUCGGUG-3'; hsa-miR-15a-5p inhibitor, 5'-CAC AAACCAUUAUGUGCUGCUA-3'; mimic NC, 5'-AAUUCG UAGCUUGCAUGCAAGC-3' and inhibitor NC, 5'-CAGUAC UUUGUGUAGUACAA-3'.

Overexpression of CCND1 in HT29 cells. The CCND1 cDNA sequence was cloned into pcDNA 3.1 vector to upregulate its expression. Plasmids (pcDNA3.1-CCND1 and pcDNA 3.1) were purchased from OBiO Technology (Shanghai) Corp., Ltd. For plasmid transfection, HT29 cells were seeded into 6 -well plates $\left(1 \times 10^{5}\right.$ cells/well). Cells were cultured to $80 \%$ confluence, and either pcDNA3.1 vector $(2.5 \mu \mathrm{g})$ or pcDNA3.1-CCND1 $(2.5 \mu \mathrm{g})$ was transfected using Lipofectamine 2000 (Invitrogen; Thermo Fisher Scientific, Inc.) at $37^{\circ} \mathrm{C}$ according to the manufacturer's instructions. Cells were cultured in DMEM for $6 \mathrm{~h}$ at $37^{\circ} \mathrm{C}$. Subsequent experiments were performed $48 \mathrm{~h}$ post-transfection. pcDNA 3.1 vector $(\mathrm{NC})$ was used as a negative control.

$R N A$ extraction and reverse transcription-quantitative $P C R$ $(R T-q P C R)$. Cells were harvested and total RNA was extracted using TRIzol ${ }^{\circledR}$ reagent (Invitrogen; Thermo Fisher Scientific, Inc.). RNA concentration was quantified using a NanoDrop ${ }^{\mathrm{TM}}$ spectrophotometer (NanoDrop Technologies; Thermo Fisher Scientific, Inc.). RNA (1 $\mu \mathrm{g})$ was reverse-transcribed at $37^{\circ} \mathrm{C}$ for $15 \mathrm{~min}$ and $85^{\circ} \mathrm{C}$ for $5 \mathrm{sec}$ using the PrimeScript Reverse Transcriptase system (Takara Biotechnology Co., Ltd.). The qPCR amplification was performed using SYBR Green Master Mix (Takara Biotechnology Co., Ltd.) under the following conditions: Initial denaturation at $95^{\circ} \mathrm{C}$ for $10 \mathrm{~min}$; followed by 40 cycles at $94^{\circ} \mathrm{C}$ for $15 \mathrm{sec}, 55^{\circ} \mathrm{C}$ for $30 \mathrm{sec}$ and $70^{\circ} \mathrm{C}$ for $30 \mathrm{sec}$. The $2^{-\mathrm{DDCq}}$ method was employed to calculate the relative expression levels and U6 was taken as an endogenous control to normalize miR-15a-5p expression (16). The following primers were used for PCR: miR-15a-5p forward (F), 5'-TAGCAGCACATAATGGTTTGTG-3' and reverse (R), 5'-GAACATGTCTGCGTATCTCAC-3'; CCND1 F, 5'-GGC GGAGGAGAACAAACA-3' and R, 5'-ATGCAGGGCGGA TTGGAAA-3'; $\beta$-actin F, 5'-CCTCGCCTTTGCCGATCC-3' and R, 5'-GGATCTTCATGAGGTAGTCAGTC-3'; and U6 F, 5'-CTCGCTTCGGCAGCAC-3' and R, 5'-ACGCTTCACGAA TTTGCGT-3'.

Western blot assay. Total protein from HT-29 cells was extracted using a RIPA kit (Beyotime Institute of Biotechnology) with the addition of $1 \%$ proteinase inhibitor. Protein concentrations were detected via the BCA method (Beyotime Institute 
of Biotechnology). After $5 \mathrm{~min}$ heating at $95^{\circ} \mathrm{C}$, proteins from each sample were separated by $10 \%$ SDS-PAGE (Nanjing KeyGen Biotech Co., Ltd.) using $20 \mu \mathrm{g}$ per lane, then transferred onto a $0.22 \mu \mathrm{m}$ PVDF membrane (Beyotime Institute of Biotechnology). The proteins were blocked with $5 \%$ non-fat milk at room temperature for $2 \mathrm{~h}$, then protein bands were incubated with primary antibodies overnight at $4^{\circ} \mathrm{C}$. Next, the protein bands were washed with TBST (TBS buffer with $0.1 \%$ Tween 20) three times and incubated with a horseradish peroxidase (HRP)-conjugated goat anti-rabbit secondary IgG (1:5,000; cat. no. ab6721; Abcam) for $1 \mathrm{~h}$ at room temperature. The protein bands were washed with TBST an additional four times and visualized using an ECL kit (Bio-Rad Laboratories, Inc. and observed using Image Lab software 4.0 (Bio-Rad Laboratories, Inc.). Primary antibodies against CCND1 (cat. no. ab40754, 1:5,000) and $\beta$-actin (cat. no. ab8227, $1: 5,000)$ were purchased from Abcam. $\beta$-actin was used as an endogenous control to normalize CCND1 expression.

5-Ethynyl-2'-deoxyuridine (EdU) assay. The cellular proliferation rate was measured using an EdU assay. A total of $1 \times 10^{5}$ cells/well were plated into 24 -well plates and left to adhere overnight. Following transfection, the cells were incubated with $100 \mu \mathrm{l} /$ well EdU (Nanjing KeyGen Biotech Co.,Ltd.) for $2 \mathrm{~h}$, fixed with $4 \%$ paraformaldehyde for $30 \mathrm{~min}$ at room temperature and incubated with DAPI solution to counterstain cell nuclei for 15-30 min at room temperature. Proliferative cells were determined under a fluorescence microscope (Leica Microsystems $\mathrm{GmbH}$ ) at 400x magnification.

Cell Counting Kit (CCK)-8 assay. A CCK-8 assay was performed to assess cell viability. HT29 cells were seeded into a 96-well plate at a density of $1 \times 10^{5}$ cells/well. The detection was performed at $0,24,48$ and $72 \mathrm{~h}$. After adding $10 \mu \mathrm{l} \mathrm{CCK}-8$ reagent (Nanjing Jiancheng Bioengineering Institute) per well and incubating for $2 \mathrm{~h}$, the absorbance at $450 \mathrm{~nm}$ was measured using a Multiscan FC plate reader and analyzed with SkanIt for Multiscan FC 3.1 software (Thermo Fisher Scientific, Inc.).

Wound scratch assay. The HT29 cells ( $1 \times 10^{5}$ cells/well) cultured in a 6-well plate were grown to form a monolayer. Meanwhile, in order to inhibit cell proliferation, the monolayer of cells was treated with $5 \mu \mathrm{M}$ mitomycin-C (Sigma-Aldrich; Merck KGaA) for $2 \mathrm{~h}$. Following incubation for $48 \mathrm{~h}$ at $37^{\circ} \mathrm{C}$ to $90 \%$ confluence, cells were scratched using a $100-\mu 1$ pipette tip. The cells were cultured in 10\% FBS-free DMEM at $37^{\circ} \mathrm{C}$ and washed twice using PBS. Images were captured at 0 and $48 \mathrm{~h}$ after scratching using a light microscope at x100 magnification (Olympus Corporation). The wound zone distances were measured using Image J 1.51 software (National Institutes of Health).

Transwell assay. Cell migration and invasion assays were performed using 24-well Transwell chambers (Nanjing KeyGen Biotech Co., Ltd.). For the invasion assay, the Transwell chambers were precoated with Matrigel (Nanjing KeyGen Biotech Co., Ltd.) for $6 \mathrm{~h}$ at $37^{\circ} \mathrm{C}$. A total of $1 \times 10^{6}$ cells in $100 \mu \mathrm{l}$ serum-free DMEM were plated in the upper chamber and $500 \mu 1$ medium supplemented with $10 \%$ FBS was used in the bottom chambers as chemoattractant. The cells were incubated at $37^{\circ} \mathrm{C}$ and $5 \% \mathrm{CO}_{2}$ for $24 \mathrm{~h}$. The migrated and invaded cells on the reverse side of the chamber inserts were fixed with $4 \%$ polyoxymethylene (Sigma-Aldrich; Merck $\mathrm{KGaA}$ ) for $30 \mathrm{~min}$ at $25^{\circ} \mathrm{C}$ and stained with $0.1 \%$ crystal violet (Sigma-Aldrich; Merck $\mathrm{KGaA}$ ) for $15 \mathrm{~min}$ at $25^{\circ} \mathrm{C}$. The number of cells was measured in five randomly selected fields of view using a light microscope (IX73, Olympus Corporation; magnification, $\mathrm{x} 200)$.

Dual-luciferase reporter assay. The target gene of hsa-miR-15a-5p was analyzed using bioinformatics to verify whether CCND1 was the direct target gene of hsa-miR-15a-5p.

The amplified 3'-UTR fragments of CCND1 were cloned into the pmirGLO luciferase reporter vector (Promega Corporation). The HT29 cells were seeded in 24-well plates at a density of $1 \times 10^{5}$ cells/well with DMEM supplemented with $10 \%$ FBS. Subsequently, cells were transfected with $50 \mathrm{nM}$ hsa-miR-15a-5p mimics (5'-UAGCAGAUCCCAAUG GUCGGUG-3'), $100 \mathrm{nM}$ mimic negative control (5'-AAU UCGUAGCUUGCAUGCAAGC-3') and $0.5 \mu \mathrm{g}$ pmirGLO luciferase reporter vector containing the wild type (WT) or mutant (Mut) 3'-UTR sequences of CCND1. Transfection were performed using Lipofectamine 2000 (Invitrogen; Thermo Fisher Scientific, Inc.) at $37^{\circ} \mathrm{C}$ according to the manufacturer's instructions. At $48 \mathrm{~h}$ post-transfection, the luciferase activity was assessed by dual-luciferase reporter assay system (Promega Corporation). Firefly luciferase activities were normalized to Renilla luciferase activities.

Statistical analysis. All data were analyzed using SPSS 19.0 statistical software (IBM Corp.). Data are expressed as the mean $\pm S D(n=3)$. Differences between the paired tissue samples from patients in Fig. 1A were analyzed using a paired Student's t-test, while comparisons between two groups were determined using an unpaired Student's t-test for unpaired samples. Comparisons among multiple groups were analyzed by one-way ANOVA followed by Tukey's post hoc test. $\mathrm{P}<0.05$ was considered to indicate a statistically significant difference.

\section{Results}

hsa-miR-15a-5p is decreased in colon tumor tissues and cell lines. RT-qPCR analysis was performed to detect hsa-miR-15a-5p expression levels in colon carcinoma. The results showed that hsa-miR-15a-5p expression was significantly decreased in colon carcinoma tissues compared with adjacent normal tissues (Fig. 1A). Likewise, hsa-miR-15a-5p expression levels were significantly downregulated in human colon carcinoma cell lines (SW480 and HCT116) and human colorectal cancer cell line (HT29) compared with the human normal colonic epithelial cell line NCM460, especially in HT29 cells (Fig. 1B). Hence, HT29 cells were chosen for subsequent experiments.

hsa-miR-15a-5p overexpression inhibits proliferation, migration and invasion of colon cancer cells. To further investigate the role of hsa-miR-15a-5p in the regulation of colon cancer, hsa-miR-15a-5p was artificially overexpressed in HT29 cells. As shown in Fig. 2A, the results showed that hsa-miR-15a-5p expression was increased following transfection 
A

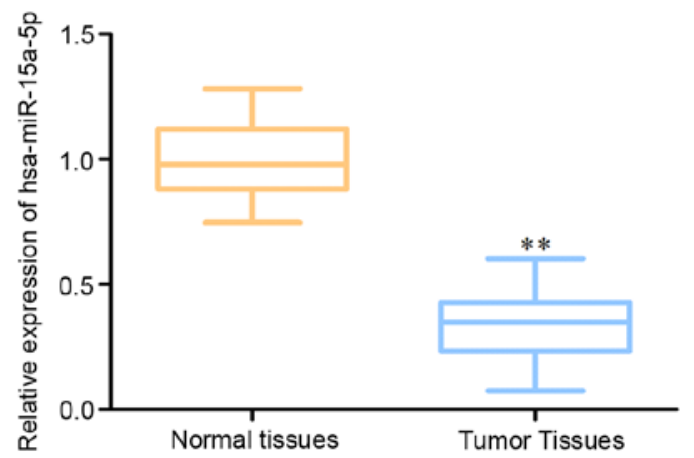

B

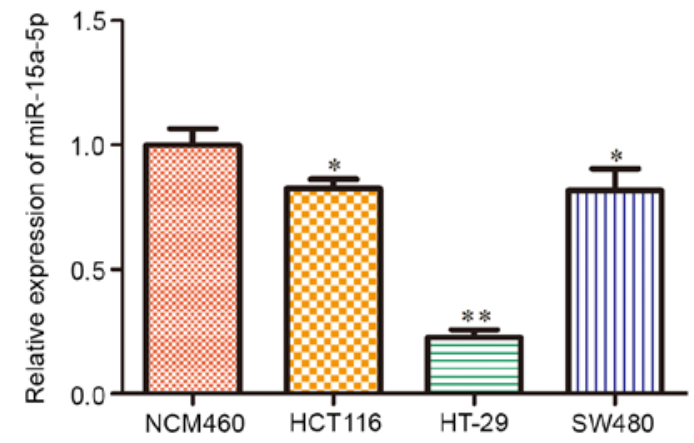

Figure 1. hsa-miR-15a-5p is decreased in colon tumor tissues and cell lines. (A) Relative expression levels of hsa-miR-15a-5p in colon cancer and normal tissues were detected via RT-qPCR. (B) Relative expression levels of hsa-miR-15a-5p in different colon cancer cell lines were detected via RT-qPCR. Data are presented as the mean \pm standard deviation and analyzed using a Student's t-test. ${ }^{*} \mathrm{P}<0.05$ and ${ }^{* *} \mathrm{P}<0.01$ vs. normal tissues or normal cells. miR, microRNA; RT-qPCR, reverse transcription-quantitative PCR.
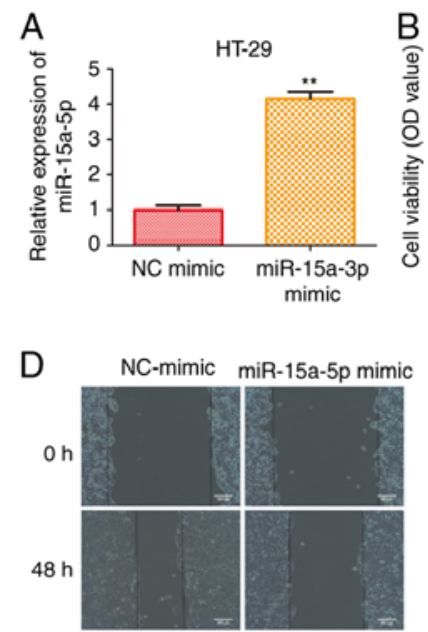
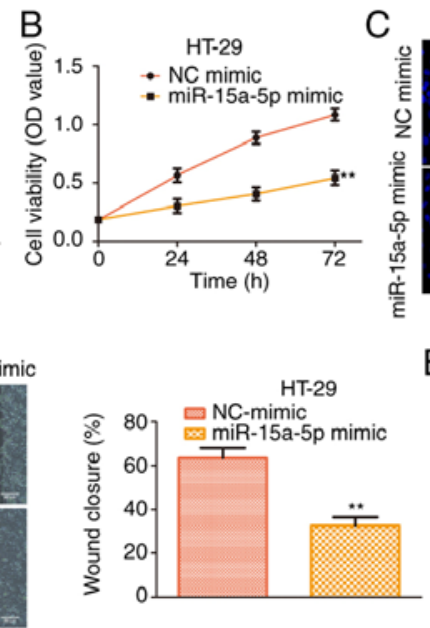

$\mathrm{F}$
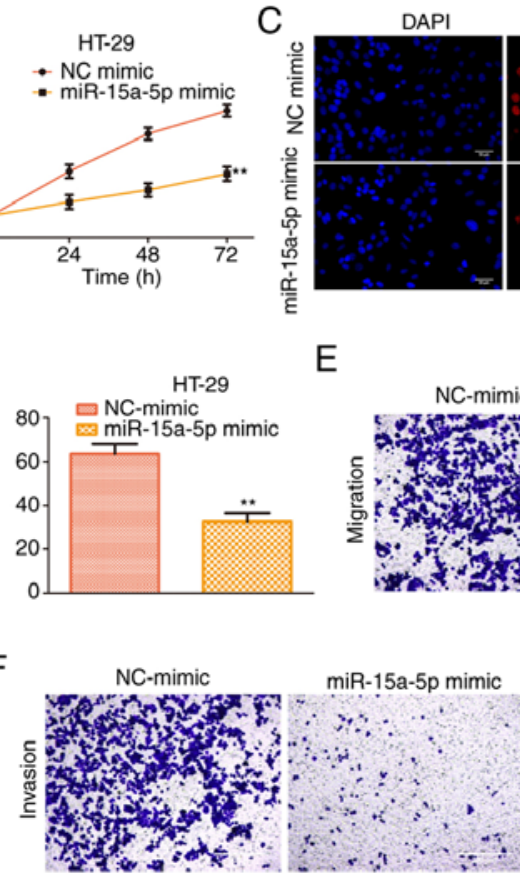

$\mathrm{E}$
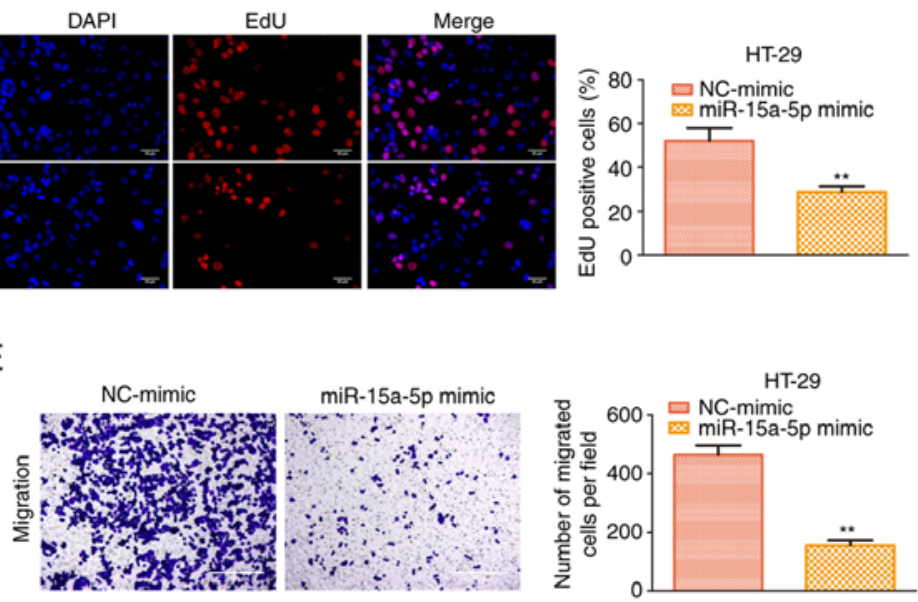

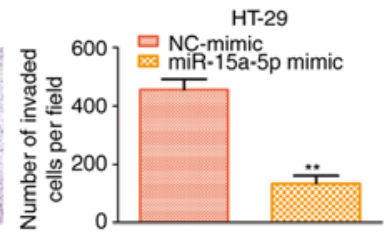

Figure 2. hsa-miR-15a-5p overexpression inhibits the proliferation, migration and invasion of colon cancer cells. HT29 cells were transfected with miR-15a-5p mimic or NC mimic. (A) Reverse transcription-quantitative PCR analysis of the transfection efficiency of hsa-miR-15a-5p mimic. (B) Cell viability was measured using a Cell Counting Kit-8 assay. (C) Determination of cell proliferation using an EdU incorporation assay. Scale bar, $50 \mu$ m. (D) Representative images and quantitative data of scratch wound closure assay. Scale bar, $200 \mu \mathrm{m}$. (E) Representative images and quantitative data of Transwell migration assay. Scale bar, $200 \mu \mathrm{m}$. (F) Representative images and quantitative data of Transwell invasion assay. Scale bar, $200 \mu \mathrm{m}$. Data are presented as the mean \pm standard deviation and analyzed using Student's t-test. ${ }^{* *} \mathrm{P}<0.01$ vs. NC mimic. miR, microRNA; NC, negative control; EdU, 5-ethynyl-2'-deoxyuridine.

with the hsa-miR-15a-5p mimic. CCK-8 and EdU incorporation assays revealed that hsa-miR-15a-5p overexpression significantly inhibited the cell viability and proliferative activity of HT29 cells (Fig. 2B and C). In line with the aforementioned results, hsa-miR-15a-5p repressed cell migration and invasion of HT29 cells (Fig. 2D-F). These findings suggested that the proliferative, migratory and invasive abilities of HT29 cells were reduced by hsa-miR-15a-5p overexpression.

hsa-miR-15a-5p directly targets the CCNDI gene. To investigate the underlying mechanism of hsa-miR-15a-5p in regulating colon cancer progression in vitro, the present study first predicted the target gene of hsa-miR-15a-5p using multiple bioinformatics analyses. ENCORI (starbase.sysu.edu. $\mathrm{cn} /$ ), TargetScan (targetscan.org/mamm_31/) and miRWalk (mirwalk.umm.uni-heidelberg.de/) were used to predict and screen the putative target of has-miR-15a-5p. As shown in Fig. 3A, 159 potential targets were clustered, and CCND1 was considered as the potential target since it is a well-known cancer-associated gene (17). To verify whether hsa-miR-15a-5p binds to the CCND1 gene, the present study designed luciferase reporter plasmids containing WT CCND1 3'-UTR or hsa-miR-15a-5p binding site mutated at the CCND1 3'-UTR (Fig. 3B). As presented in Fig. 3C, hsa-miR-15a-5p overexpression decreased the luciferase activity of WT CCND1 3'-UTR, whereas a mutation at the hsa-miR-15a-5p binding site in the 

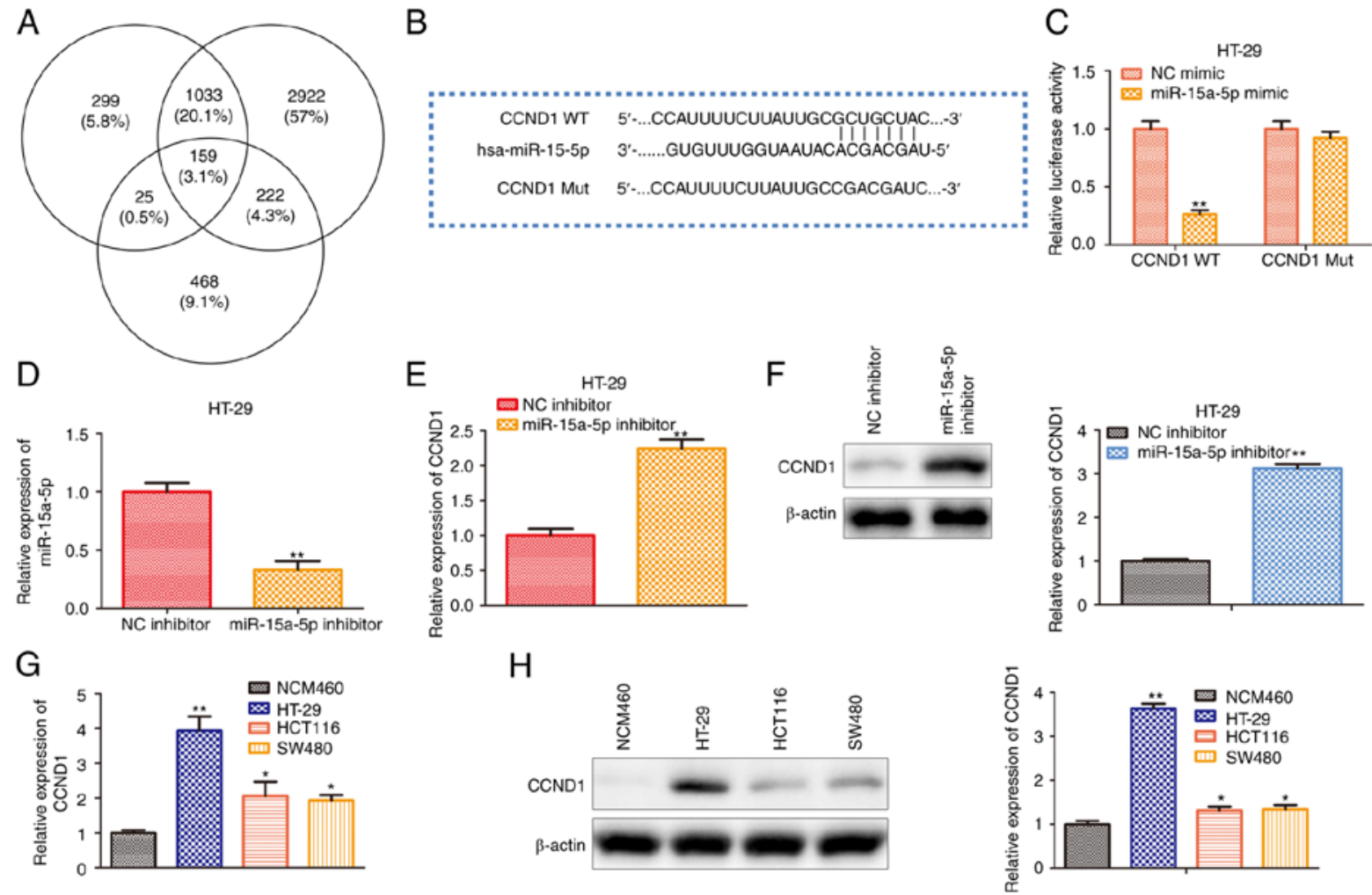

Figure 3. hsa-miR-15a-5p directly targets the CCND1 gene. (A) Schematic presentation of hsa-miR-15a-5p target genes. (B) hsa-miR-15a-5p binding site on the 3'-UTR of CCND1 mRNA. (C) Dual-luciferase reporter assays in HT29 cells. (D) Reverse transcription-quantitative PCR analysis of the transfection efficiency of hsa-miR-15a-5p inhibitor. (E) mRNA expression levels of CCND1 in hsa-miR-15a-5p-knockdown HT29 cells. (F) Protein expression levels of CCND1 in hsa-miR-15a-5p-knockdown HT29 cells. (G) mRNA expression of CCND1 in colon cancer cells. (H) Protein expression of CCND1 in colon cancer cells. Data are presented as the mean \pm standard deviation. ${ }^{*} \mathrm{P}<0.05$ and ${ }^{* *} \mathrm{P}<0.01$ vs. NC group or normal tissues/cells. miR, microRNA; NC, negative control; CCND1, G1/S-specific cyclin-D1; UTR, untranslated region; WT, wild-type; Mut, mutant.

3'-UTR of CCND1 almost completely abolished the repressive effects of hsa-miR-15a-5p overexpression on luciferase activity. Moreover, the expression levels of hsa-miR-15a-5p in HT29 cells transfected with hsa-miR-15a-5p inhibitor were assessed. It was found that the expression of hsa-miR-15a-5p was reduced (Fig. 3D). Meanwhile, RT-qPCR and western blotting indicated that hsa-miR-15a-5p repressed CCND1 expression at both transcriptional and translational levels (Fig. 3E and F). In addition, to estimate the role of CCND1 in colon cancer, the present study examined the expression levels of CCND1 in human normal colonic epithelial cell line NCM460, human colon carcinoma cell lines (SW480 and HCT116) and human colon adenocarcinoma cell line (HT29) by RT-qPCR and western blotting. Compared with the NCM460 cell line, the mRNA and protein expression levels of CCND1 in colon cancer cell lines were significantly increased, especially in the HT29 cell line (Fig. 3G and H).

CCDN1 overexpression promotes colon cancer progression. To investigate the function of CCDN1 in colon cancer progression, pcDNA-control and pcDNA-CCDN1 were transfected into HT29 cells. CCK-8 and EdU incorporation assays revealed that CCDN1 overexpression significantly promoted the cell viability and proliferative activity of HT29 cells (Fig. 4A and B). In line with the aforementioned results, CCDN1 overexpression promoted cell migration and invasion of HT29 cells (Fig. 4C and D). Therefore, CCDN1 overexpression could promote colon cancer progression.
hsa-miR-15a-5p inhibits oncogenic progression of colon cancer via targeting the CCNDI gene. To confirm whether the effects of hsa-miR-15a-5p on colon cancer cells were mediated by CCND1, CCND1 was overexpressed in HT29 cells transfected with hsa-miR-15a-5p mimic. As shown in Fig. 5A, the expression of CCND1 was increased following transfection with pcDNA-CCND1 compared with pcDNA-control in HT29 cells. CCK-8 and EdU assays indicated that the proliferative capacity of HT29 cells was inhibited by transfection with a hsa-miR-15a-5p mimic, while this was promoted following CCND1 overexpression (Fig. 5B and C). Similar to the results of cell proliferation assays, CCND1 overexpression partially abolished the inhibitory effects of hsa-miR-15a-5p on HT29 cell migration and invasion (Fig. 5D-F). These results suggested that CCND1 functions as a mediatory factor in relaying hsa-miR-15a-5p signaling to colon cancer progression in vitro.

\section{Discussion}

Colon carcinoma is the third most common malignancy and the fourth leading cause of cancer mortality worldwide (1). It has been well-established that aging, the environment, a high-fat diet and heredity factors are key risk factors for colon carcinoma (2-4). The majority of patients suffer from poor prognosis due to the high recurrence of colon cancer (18). Hence, understanding the underlying molecular mechanism of colon cancer is of importance. 
A

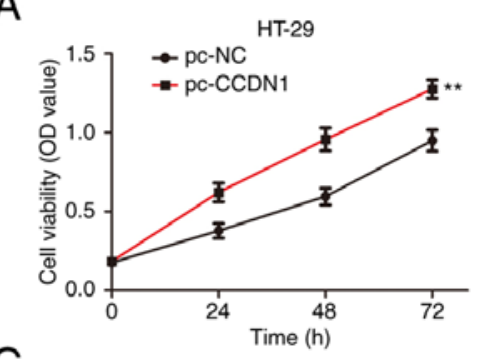

B

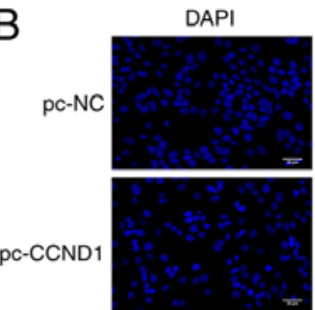

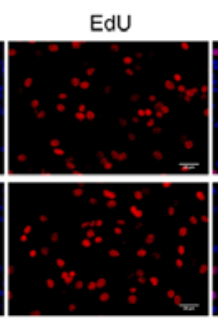
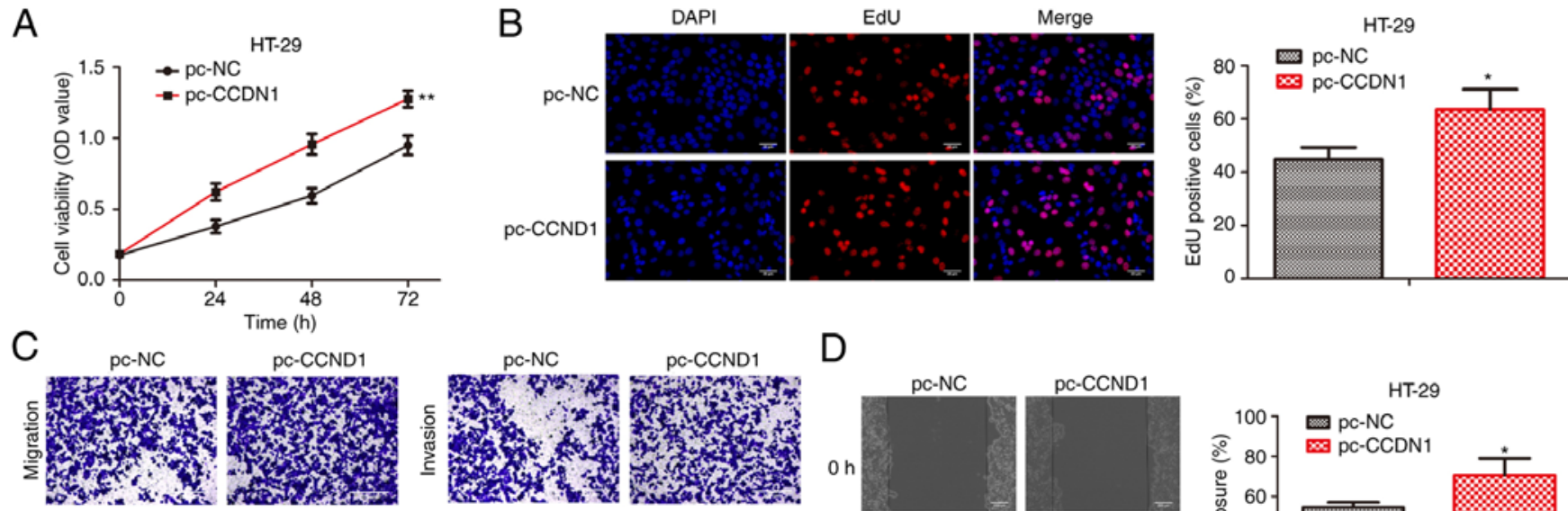

$\mathrm{D}$
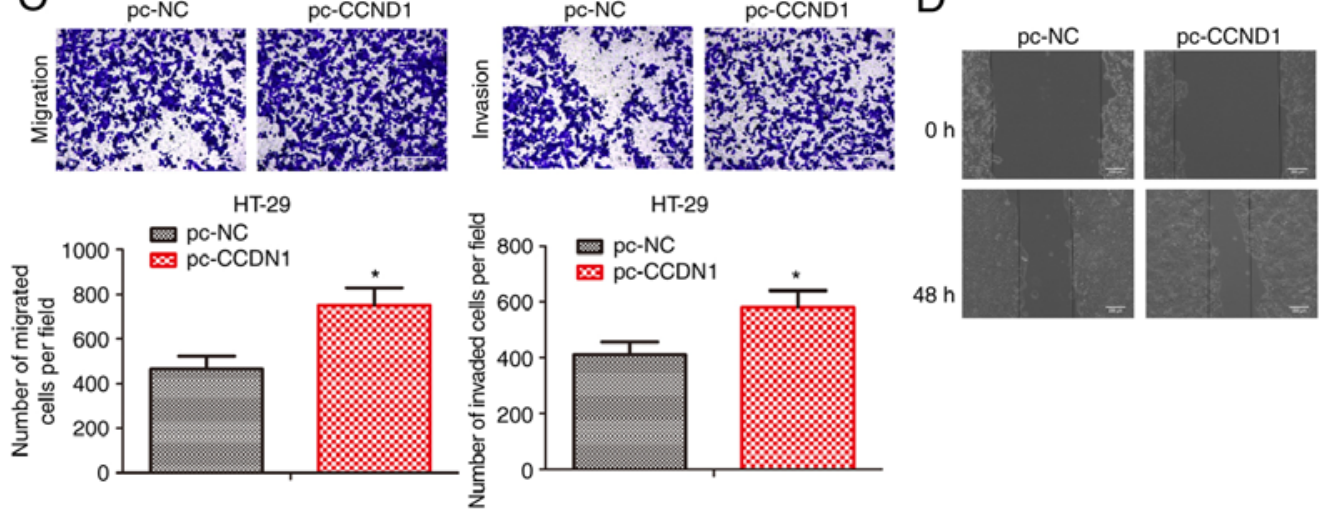

$48 \mathrm{~h}$
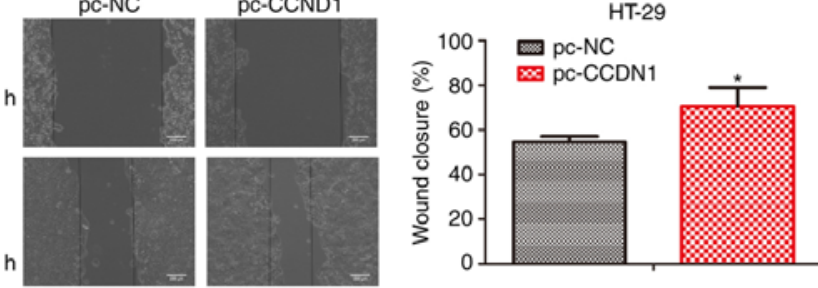

Figure 4. Overexpression of CCDN1 promotes the progression of colon cancer. HT29 cells were transfected with pc-CCDN1 or pc-NC. (A) Cell viability measured using a Cell Counting Kit-8 assay. (B) Determination of cell proliferation using an EdU incorporation assay. Scale bar, $50 \mu \mathrm{m}$. (C) Representative images and quantitative data of Transwell migration and invasion assays. Scale bar, $200 \mu \mathrm{m}$. (D) Representative images and quantitative data of scratch wound closure assay. Scale bar, $200 \mu \mathrm{m}$. Data are presented as the mean \pm standard deviation. ${ }^{*} \mathrm{P}<0.05$ and ${ }^{* *} \mathrm{P}<0.01$ vs. pc-NC. NC, negative control; CCND1, G1/S-specific cyclin-D1; EdU, 5-ethynyl-2'-deoxyuridine.

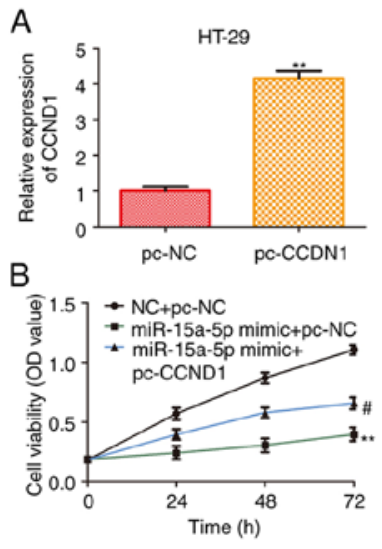

C
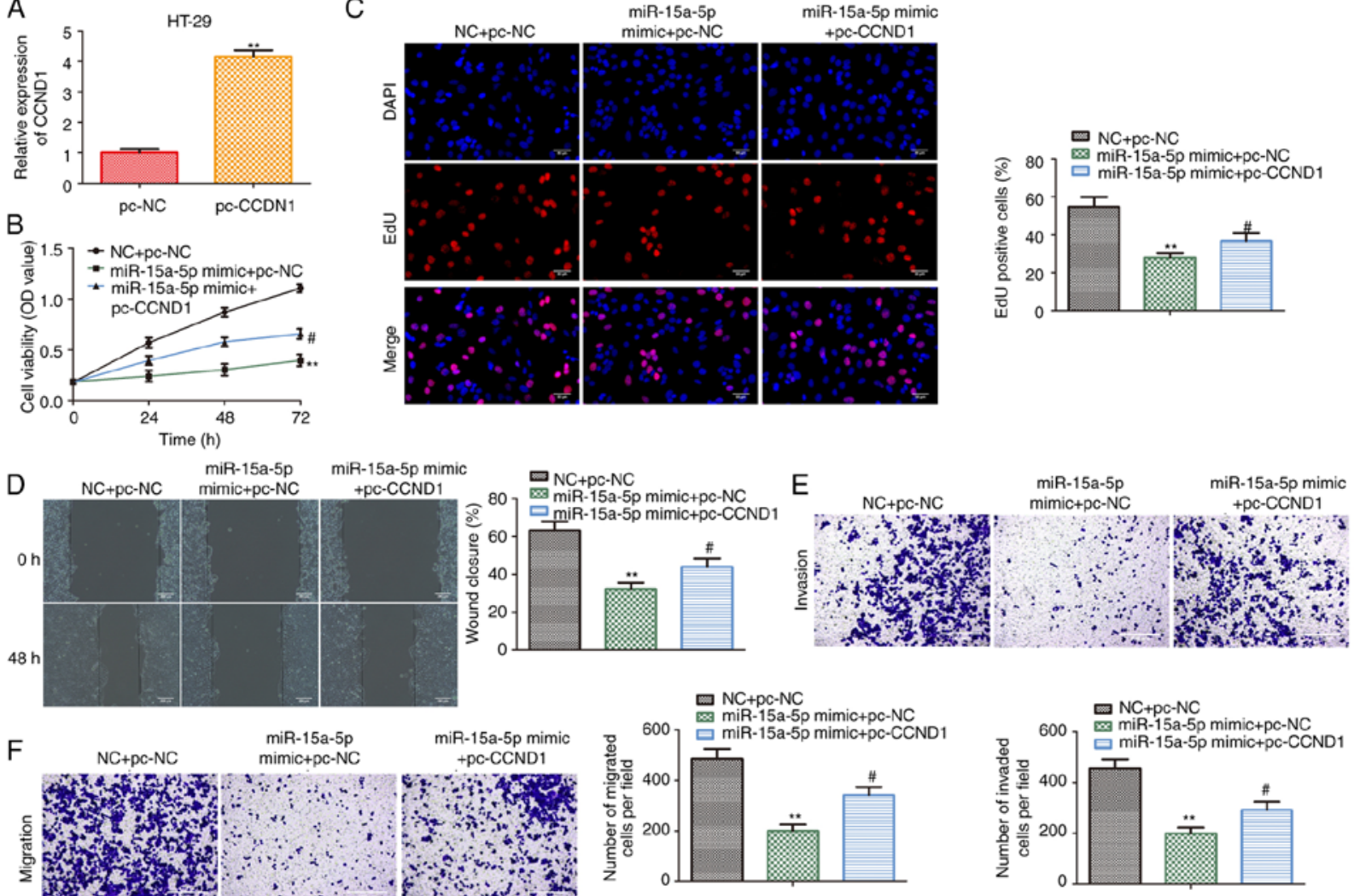

Figure 5. hsa-miR-15a-5p represses oncogenic progression via targeting CCND1. HT29 cells were co-transfected with hsa-miR-15a-5p with pc-CCDN1 or pc-NC for $48 \mathrm{~h}$. (A) Reverse transcription-quantitative PCR analysis of the transfection efficiency of pc-CCND1. (B) Cell Counting Kit-8 assay was performed to measure cell viability. (C) Determination of cell proliferation using an EdU incorporation assay. Scale bar, $50 \mu \mathrm{m}$. (D) Representative images and quantitative data of scratch wound closure assay. Scale bar, $200 \mu \mathrm{m}$. (E) Representative images and quantitative data of Transwell invasion assay. Scale bar, $200 \mu \mathrm{m}$. (F) Representative images and quantitative data of Transwell migration assay. Scale bar, $200 \mu \mathrm{m}$. Data are presented as the mean \pm standard deviation and were analyzed using one-way ANOVA. ${ }^{*} \mathrm{P}<0.05,{ }^{"} \mathrm{P}<0.05$ and ${ }^{* *} \mathrm{P}<0.01$ v. NC + pc-NC. miRNA, microRNA; NC, negative control; CCND1, G1/S-specific cyclin-D1; EdU, 5-ethynyl-2'-deoxyuridine. 
In the past decades, numerous studies have reported that miRNAs act as regulatory factors in cancer progression and metastasis via targeting crucial genes $(19,20)$. Identification of miRNAs with key roles in targeting and regulating genes in signaling and metabolic pathways is a novel approach for cancer treatment (21). Of note, hsa-miR-15a-5p is reported to be closely associated with colorectal cancer survival (15). miR-15a-5p is part of the miR-15/16 cluster on chromosome 13q14.3, which acts as a tumor suppressor of chronic lymphocytic lymphoma by promoting apoptosis via targeting BCL2 (22). The present study found that hsa-miR-15a-5p expression was significantly downregulated in colon tumor tissues and cell lines. To further investigate the effects of hsa-miR-15a-5p on colon cancer, hsa-miR-15a-5p was overexpressed in HT29 cells via hsa-miR-15a-5p mimic transfection. The present study found a negative regulatory effect of hsa-miR-15a-5p overexpression on colon cancer cell proliferation, migration and invasion. Therefore, similar to its function in chronic lymphocytic lymphoma, hsa-miR-15a-5p acts as a tumor suppressor in colon cancer.

Multiple bioinformatics analyses predicted CCND1 was a potential target of hsa-miR-15a-5p. CCND1 has emerged as a key regulator of cell cycle progression by interacting with CDKs during the G1 to S-phase transition (17). Dysregulation of the cell cycle is the most typical cause of carcinomas, while CCND1 expression is upregulated in various cancers, including non-small cell lung cancer, pancreatic ductal adenocarcinoma and ovarian cancer, resulting in abnormalities of cell proliferation (23-25). More importantly, it has also been found to induce malignant phenotypes by promoting cell migration and metastasis $(26,27)$. The present study demonstrated that CCND1 was the direct target of hsa-miR-15a-5p using a dual-luciferase reporter assay. In addition, CCND1 expression was upregulated, and negatively regulated by hsa-miR-15a-5p in colon cancer cells, which was confirmed by RT-qPCR and western blot analyses. Consistently, CCND1 was correspondingly highly expressed in colon cancer tissues and cell lines. To further determine the role of CCND1 in colon cancer, CCND1 was artificially overexpressed in HT29 cells, and it was found that CCND1 overexpression partially hindered the repressive effects of hsa-miR-15a-5p on the proliferation, migration and invasion of colon cancer cells.

In summary, the present study found that hsa-miR-15a-5p expression was significantly decreased in clinical colon tumor samples and cell lines. The present study provided evidence that hsa-miR-15a-5p may act as a tumor suppressor of colon cancer by targeting CCND1. These findings provided an innovative target and a potential for the treatment and prognosis of colon cancer.

\section{Acknowledgements}

Not applicable.

\section{Funding}

This work was supported by the Hospital-level topic of Jiangsu Cancer Hospital (grant no. ZM202011) and The Sixth Batch of National Heritage Studios of Traditional Chinese Medicine Experts [Education and Development of Traditional Chinese Medicine; grant no. (2017) 29].

\section{Availability of data and materials}

The datasets used and/or analyzed during the current study are available from the corresponding author on reasonable request.

\section{Authors' contributions}

$\mathrm{ZL}, \mathrm{ZZ}, \mathrm{XZ}$ and $\mathrm{YB}$ conceived and designed the study and methodology. ZL, ZZ, YaW, YiW, WL and ZW performed the experiments and collected the data. $\mathrm{ZL}, \mathrm{ZZ}$ and $\mathrm{YaW}$ analyzed and interpreted the data. ZL, XZ and YB confirm the authenticity of all the raw data. $\mathrm{ZL}$ and $\mathrm{ZZ}$ drafted the manuscript. XZ and YB revised the manuscript. All authors read and approved the final manuscript.

\section{Ethics approval and consent to participate}

The present study was approved by the Medical Ethics Committee of the Affiliated Cancer Hospital of Nanjing Medical University and Jiangsu Cancer Hospital and Jiangsu Institute of Cancer Research [(2016) approval no. 231]. All participants provided written informed consent.

\section{Patient consent for publication}

Not applicable.

\section{Competing interests}

The authors declare that they have no competing interests.

\section{References}

1. Bray F, Ferlay J, Soerjomataram I, Siegel RL, Torre LA and Jemal A: Global cancer statistics 2018: GLOBOCAN estimates of incidence and mortality worldwide for 36 cancers in 185 countries. CA Cancer J Clin 68: 394-424, 2018.

2. Itatani Y,Kawada K,FujishitaT,KakizakiF,HiraiH,MatsumotoT, Iwamoto M, Inamoto S, Hatano E, Hasegawa S, et al: Loss of SMAD4 from colorectal cancer cells promotes CCL15 expression to recruit $\mathrm{CCR} 1^{+}$myeloid cells and facilitate liver metastasis. Gastroenterology 145: 1064-1075.e11, 2013.

3. Aghakhani A, Hamkar R, Ramezani A, Bidari-Zerehpoosh F, Sabeti S, Ghavami N, Banifazl M, Rashidi N and Eslamifar A: Lack of human papillomavirus DNA in colon adenocarcinama and adenoma. J Cancer Res Ther 10: 531-534, 2014.

4. Zhang Y, Lin C, Liao G, Liu S, Ding J, Tang F, Wang Z, Liang X, Li B, Wei Y, et al: MicroRNA-506 suppresses tumor proliferation and metastasis in colon cancer by directly targeting the oncogene EZH2. Oncotarget 6: 32586-32601, 2015.

5. Chen L, Wang J, Fu L, Zhang B, Zhang H and Ye B: Prognostic significance of metastasis associated in colon cancer 1 (MACC1) expression in patients with gallbladder cancer. J Cancer Res Ther 10: 1052-1056, 2014.

6. Manjelievskaia J, Brown D, McGlynn KA, Anderson W, Shriver CD and Zhu K: Chemotherapy use and survival among young and middle-aged patients with colon cancer. JAMA Surg 152: 452-459, 2017.

7. Nicoloso MS, Spizzo R, Shimizu M, Rossi S and Calin GA: MicroRNAs-the micro steering wheel of tumour metastases. Nature reviews. Cancer 9: 293-302, 2009.

8. Schetter AJ, Leung SY, Sohn JJ, Zanetti KA, Bowman ED, Yanaihara N, Yuen ST, Chan TL, Kwong DL, Au GK, et al: MicroRNA expression profiles associated with prognosis and therapeutic outcome in colon adenocarcinoma. JAMA 299: 425-436, 2008.

9. Schwarzenbach $\mathrm{H}$ : Clinical relevance of circulating, cell-free and exosomal microRNAs in plasma and serum of breast cancer patients. Oncol Res Treat 40: 423-429, 2017. 
10. Alvarez-Díaz S, Valle N, Ferrer-Mayorga G, Lombardía L, Herrera M, Domínguez O, Segura MF, Bonilla F, Hernando E and Muñoz A: MicroRNA-22 is induced by vitamin D and contributes to its antiproliferative, antimigratory and gene regulatory effects in colon cancer cells. Hum Mol Genet 21: 2157-2165, 2012.

11. Cai SD, Chen JS, Xi ZW, Zhang LJ, Niu ML and Gao ZY: MicroRNA-144 inhibits migration and proliferation in rectal cancer by downregulating ROCK-1. Mol Med Rep 12: 7396-7402, 2015.

12. Cottonham CL, Kaneko S and Xu L: miR-21 and miR-31 converge on TIAM1 to regulate migration and invasion of colon carcinoma cells. J Biol Chem 285: 35293-302, 2010.

13. Yi H, Geng L, Black A, Talmon G, Berim L and Wang J: The miR-487b-3p/GRM3/TGF $\beta$ signaling axis is an important regulator of colon cancer tumorigenesis. Oncogene 36 : 3477-3489, 2017.

14. Li X, Li Z, Zhu Y, Li Z, Yao L, Zhang L, Yuan L, Shang Y, Liu J and Li C: miR-524-5p inhibits angiogenesis through targeting WNK1 in colon cancer cells. Am J Physiol Gastrointest Liver Physiol 318: 827-839, 2020.

15. Mullany LE, Herrick JS, Sakoda LC, Samowitz W, Stevens JR, Wolff RK and Slattery ML: miRNA involvement in cell cycle regulation in colorectal cancer cases. Genes Cancer 9: 53-65, 2018.

16. Livak KJ and Schmittgen TD: Analysis of relative gene expression data using real-time quantitative PCR and the 2(-Delta Delta C(T)) method. Methods 25: 402-408, 2001.

17. Chang L, Guo R, Yuan Z, Shi H and Zhang D: LncRNA HOTAIR regulates CCND1 and CCND2 expression by sponging miR-206 in ovarian cancer. Cell Physiol Biochem 49: 1289-1303, 2018.

18. Douaiher J, Ravipati A, Grams B, Chowdhury S, Alatise O and Are C: Colorectal cancer-global burden, trends, and geographical variations. J Surg Oncol 115: 619-630, 2017.
19. Pan Y, Zhang J, Fu H and Shen L: miR-144 functions as a tumor suppressor in breast cancer through inhibiting ZEB1/2-mediated epithelial mesenchymal transition process. Onco Targets Ther 9: 6247-6255, 2016

20. Chen Q, Xia HW, Ge XJ, Zhang YC, Tang QL and Bi F: Serum miR-19a predicts resistance to FOLFOX chemotherapy in advanced colorectal cancer cases. Asian Pac J Cancer Prev 14: 7421-7426, 2013

21. Ritchie W, Rasko JE and Flamant S: MicroRNA target prediction and validation. Adv Exp Med Biolgy 774: 39-53, 2013.

22. Aqeilan RI, Calin GA and Croce CM: miR-15a and miR-16-1 in cancer: Discovery, function and future perspectives. Cell Death Differ 17: 215-220, 2010.

23. Orhan C, Bulut P, Dalay N, Ersen E and Buyru N: Downregulation of TCEAL7 expression induces CCND1 expression in non-small cell lung cancer. Mol Biol Rep 46: 5251-5256, 2019.

24. Chen G, Hu M, Qu X, Wang K and Qu Y: MicroRNA-584 directly targets CCND1 and inhibits cell proliferation and invasion in pancreatic cancer. Mol Med Rep 19: 719-726, 2019.

25. Dai J, Wei RJ, Li R, Feng JB, Yu YL and Liu PS: A study of CCND1 with epithelial ovarian cancer cell proliferation and apoptosis. Eur Rev Med Pharmacol Sci 20: 4230-4235, 2016.

26. Hao CY, Zhao S, Zhang LL and Liu D: SNHG16 promotes the progression of osteoarthritis through activating microRNA-93-5p/CCND1 axis. Eur Rev Med Pharmacol Sci 23: 9222-9229, 2019.

27. Nie M, Wang Y, Yu Z, Li X, Deng Y, Wang Y, Yang D, Li Q, Zeng $\mathrm{X}, \mathrm{Ju} \mathrm{J}$, et al: AURKB promotes gastric cancer progression via activation of CCND1 expression. Aging (Albany NY) 12: 1304-1321, 2020.

c) (i) (3) This work is licensed under a Creative Commons (c) NG No Attribution-NonCommercial-NoDerivatives 4.0 International (CC BY-NC-ND 4.0) License. 